\title{
Noninvasive Assessment of Liver Disease- Measurement of Hepatic Fibrosis Using Shear Wave Elastography (SWE)
}

\author{
Naoki Hotta \\ Department of Internal Medicine, Division of Gastroenterology, Masuko Momorial Hospital, Nagoya, Japan \\ Email: hotta4166@yahoo.co.jp
}

Received 19 June 2015; accepted 28 August 2015; published 31 August 2015

Copyright (C) 2015 by author and Scientific Research Publishing Inc.

This work is licensed under the Creative Commons Attribution International License (CC BY). http://creativecommons.org/licenses/by/4.0/ c) (i) Open Access

\begin{abstract}
The evaluation of liver fibrosis is said to be one of the factors of the liver cancer development. The noninvasive testing is considered for abdominal echography, MRI, a blood marker in various ways. We announce the evaluation as an article by liver fibrosis using the probe for the heart before, too. This time is shear wave elastography using the echo probe for the abdomen (SWE). We used and examined it in normal, chronic hepatitis patients with cirrhosis. We tried it, but, in the patients with cirrhosis, there was no examination in the change in a value after ascites time and ascitic drainage. The future examination thinks about a comparison with the liver biopsy, the comparison with the liver fibrosis marker. Also, we think that a malignancy and the benign differentiation should be possible for a liver tumor.
\end{abstract}

\section{Keywords}

\section{Shear Wave Elastography (SWE), Hepatic Fibrosis}

\section{Introduction}

The development of hepatic fibrosis in patients with liver disease is associated with an increased risk of liver cancer. Assessing the degree of hepatic fibrosis is therefore one of the most important factors in treatment planning. Liver biopsy is commonly performed to assess hepatic fibrosis, but this method is associated with complications such as hemorrhage. Recently, a number of studies on the noninvasive assessment of hepatic fibrosis have appeared in the literature. SWE was performed in a total of 31 subjects: 8 in the normal adult group, 17 in the chronic hepatitis group, and 6 in the cirrhosis group. In the present study, this method was applied to the assessment of hepatic fibrosis. The average of the five measurements was used as the SWE value. 


\section{Purpose}

The usefulness of SWE in assessing the degree of hepatic fibrosis was evaluated.

\section{Materials}

SWE was performed in a total of 31 subjects: 8 in the normal adult group (age range: 49 to 77 years, average age: 65.6 years), 17 in the chronic hepatitis group (age range: 35 to 79 years, average age: 58.2 years), and 6 in the cirrhosis group (age range: 55 to 74 years, average age: 69.1 years). The diagnosis was confirmed by liver biopsy in 7 subjects in the chronic hepatitis group and 2 subjects in the cirrhosis group. For the remaining 10 subjects in the chronic hepatitis group and the remaining 2 subjects in the cirrhosis group, the diagnosis was confirmed based on the findings obtained by diagnostic imaging studies (ultrasound and/or CT examination), clinical evaluation, and clinical laboratory tests. The subjects in the normal adult group were confirmed to have no history of liver disease before the time of entry into the study. Written informed consent to participate in the study was obtained from each subject.

\section{Subjects}

A diagnostic ultrasound system (Aplio ${ }^{\mathrm{TM}}$, Toshiba Medical Systems Corporation, Tochigi, Japan) and convex probe was employed in this study.

All SWE measurements were made by experienced abdominal radiologist (Naoki H).

SWE method that has been developed by Toshiba, a part the tissue is deformed by a push pulse, the velocity of the shear wave propagating within the tissue is detected, and the stiffness of the tissue is assessed based on the defected that shear velocity. If an obstacle such as a tumor is present in the tissue, the shear velocity in the area differs from the shear velocity in the surrounding areas. if the obstacle is stiffer than the surrounding tissues, the shear waves propagate slower. The propagating shear waves are detected by transmitting search pulses.

The curved transducer was placed intercostally at the level of the right lobe of the liver, with the target area was located in the right anterior hepatic segment at a depth of more than $2 \mathrm{~cm}$ from the hepatic capsule to avoid major vessels.

Measurement was performed five times form the right intercostal space.

The average of five measurements was used as the SWE value.

\section{Results}

The SWE values were $1.5 \mathrm{~m} / \mathrm{s}$ in the normal adult group (Figure 1), $2.73 \mathrm{~m} / \mathrm{s}$ in the chronic hepatitis group (Figure 2), $2.71 \mathrm{~m} / \mathrm{s}$ in the cirrhosis group (Figure 3), and $9.43 \mathrm{~m} / \mathrm{s}$ for patients with cirrhosis and ascites (Figure 4). In the ascites patients, we measured before and after ascetic drainage. However the change was absent in a value (Figure 5).

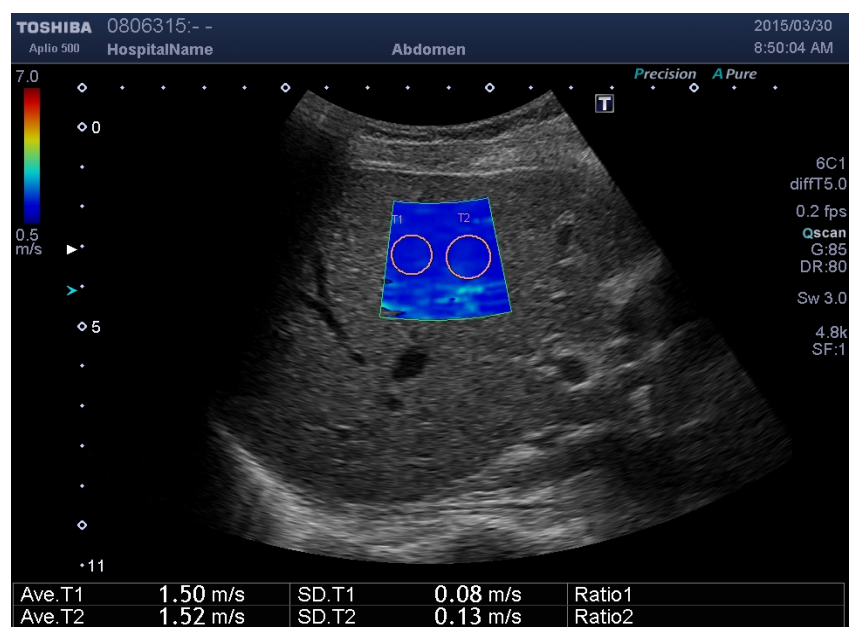

Figure 1. SWE in normal adult group. 


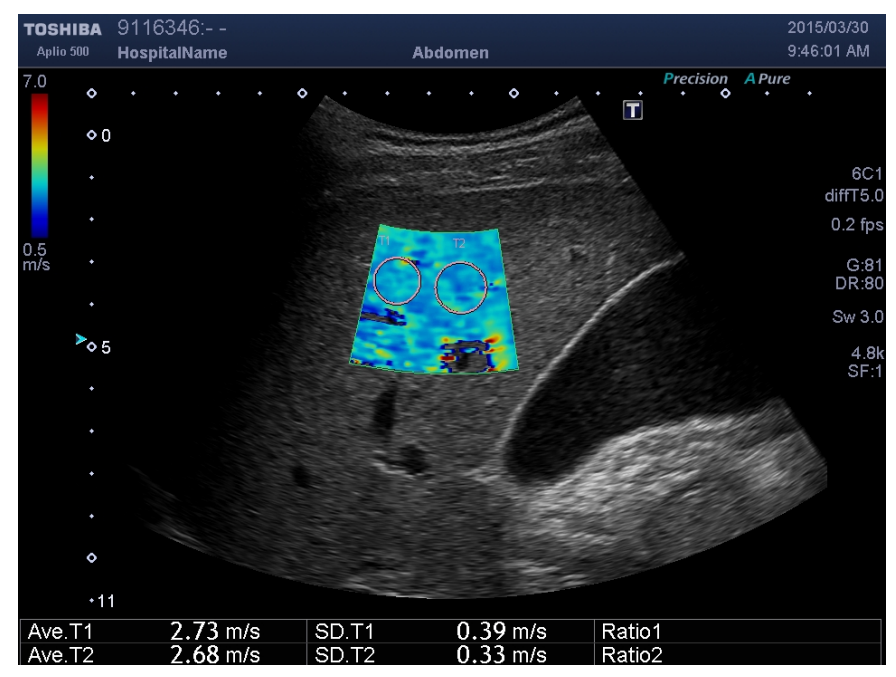

Figure 2. SWE in chronic hepatitis group.

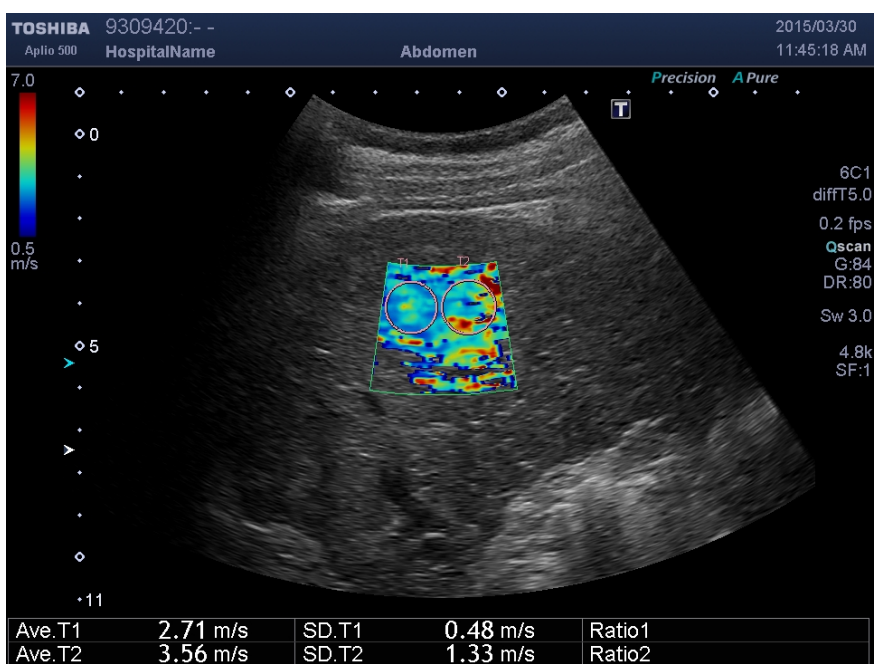

Figure 3. SWE values in cirrhosis group.

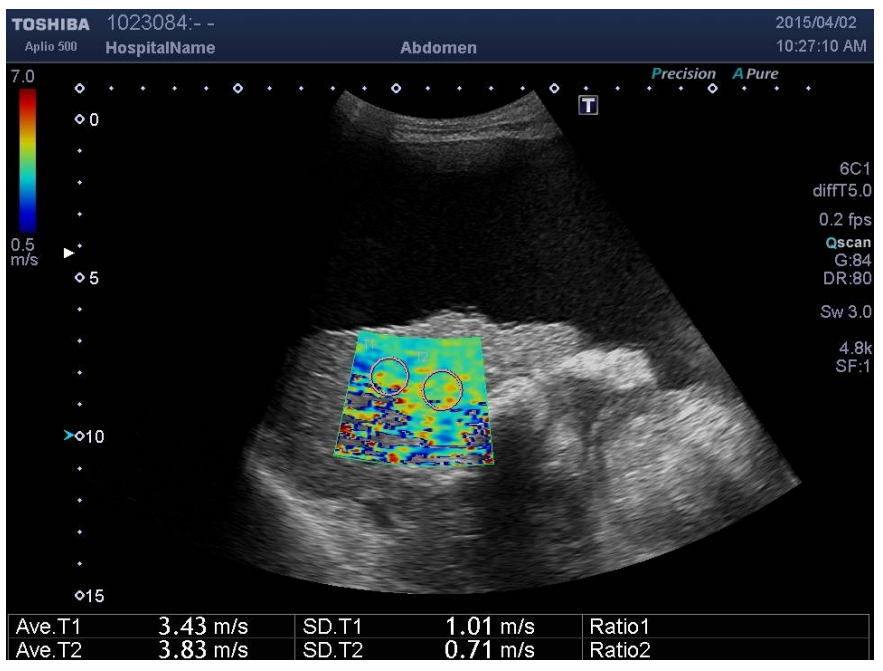

Figure 4. SWE values for patients with cirrhosis and ascites. 


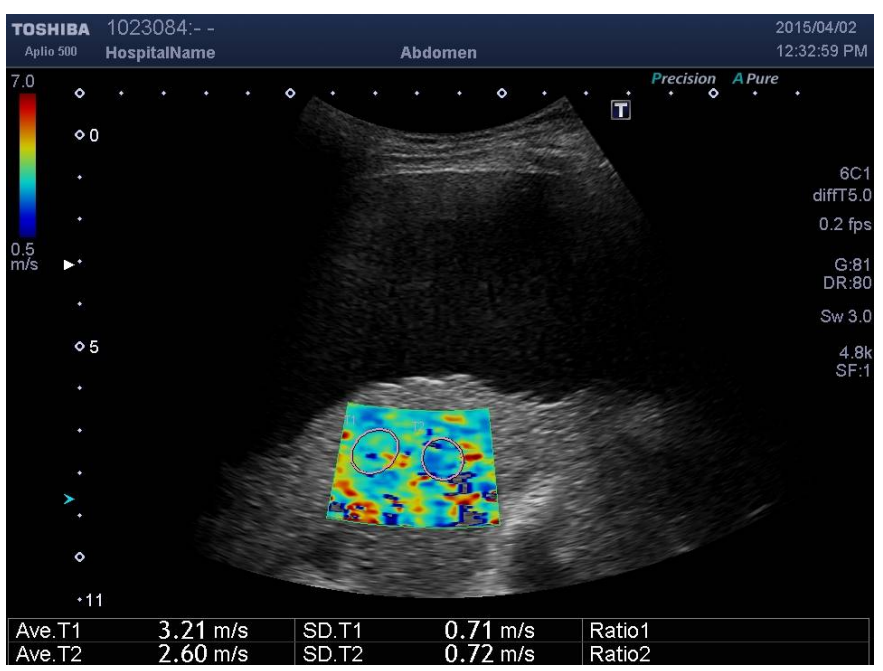

Figure 5. SWE values for patients after the ascetic drainage.

\section{Discussion}

The development of hepatic fibrosis in patients with liver disease is associated with an increased risk of liver cancer. Assessing the degree of hepatic fibrosis is therefore one of the most important factors in treatment planning. Liver biopsy is commonly performed to assess hepatic fibrosis, but this method is associated with complications such as hemorrhage. In addition, diagnostic results may vary depending on the sampling sites and the physician performing the procedure. Recently, a number of researchers have discussed the noninvasive assessment of hepatic fibrosis [1]-[3]. This method can also be applied to patients with ascites. In addition, SWE can be performed while visualizing the liver. However, several tens of minutes are needed to complete data analysis, and the patient therefore cannot be informed of the results immediately. Recently, a number of studies on the noninvasive assessment of hepatic fibrosis have appeared in the literature. Some studies have compared the results obtained by FibroScan [4], which is used to measure liver elasticity and to assess liver stiffness, with the findings of liver biopsy in patients with chronic hepatitis $\mathrm{C}$ and patients with cirrhosis. However, FibroScan is not effective in patients with ascites or in obese patients. This method is also not useful for patients with cirrhosis and severe atrophy because measurement cannot be performed while directly visualizing the liver. In the present study, SWE was found to be useful in such cases. In Fibro Test [5] [6], another noninvasive hepatic fibrosis assessment method developed in France, fibrosis is assessed based on the values determined using five assays: $\alpha 2$-macroglobulin, hepatoglobulin, $\gamma$-GTP, total bilirubin, and apolipoprotein A1. However, this method may not be suitable for routine clinical practice because analysis is expensive and, in Japan, $\alpha 2$-macroglobulin, hepatoglobulin, and apolipoprotein A1 tests are not covered by the health insurance system. In the HALT-C trial [7] [8], the degree of cirrhosis is estimated based on the platelet count, AST/ALT ratio, and INR. This method involves complicated computations and cannot differentiate between chronic hepatitis and cirrhosis. SWE is capable of such differentiation. In the guidelines on WFUMB announced in 2015, the diagnosis of SWE is equipped with, but there is not the announcement with the product made by TOSHIBA [9]. Our examination is the first time currently even if we search the data before and after the ascitic drainage of SWE in PUB Med. This examination took effect only for normal, chronic hepatitis, cirrhosis.

The future examination is an enforcement direction in comparison with hepatitis $\mathrm{C}$ before and after therapy and fibrosis, H2BPGI of the fibrosis marker new [10].

We want to examine the differentiation with a hepatocellular carcinoma and the benign tumor, the hepatocellular carcinoma before and after radiofrequency ablation [11] [12].

\section{Conclusion}

The results of the present study suggest that noninvasive SWE may become the method of choice for assessing hepatic fibrosis in routine clinical practice. We are planning to conduct a comparative study with liver biopsy regarding the differentiation between fibrosis of stage F0-F1 and higher stages, which is an important factor in 
determining whether or not treatment is required.

\section{References}

[1] Kawamoto, M., Mizuguchi, T., Katsuramaki, T., et al. (2006) Assessment of Liver Fibrosis by a Noninvasive Method of Transient Elastography and Biochemical Markers. World Journal of Gastroenterology, 12, 4325-4330.

[2] Kazemi, F., Kettaneh, A., Nkontchou, G., et al. (2006) Liver Stiffness Measurement Selects Patients with Cirrhosis at Risk of Bearing Large Oesophageal Varices. Journal of Hepatology, 45, 230-235. http://dx.doi.org/10.1016/j.jhep.2006.04.006

[3] Castera, L., Vergniol, J., Foucher, J., et al. (2005) Prospective Comparison of Transient Elastography, Fibrotest, APRI and Liver Biopsy for the Assessment of Fibrosis in Chronic Hepatitis C. Gastroenterology, 128, 343-350. http://dx.doi.org/10.1053/j.gastro.2004.11.018

[4] Ziol, M., Handra-Luca, A., Kettaneh, A., et al. (2005) Noninvasive Assessment of Liver Fibrosis by Measurement of Stiffness in Patients with Chronic Hepatitis C. Hepatology, 41, 48-54. http://dx.doi.org/10.1002/hep.20506

[5] Foucher, J., Chanteloup, E., Vergniol, J., et al. (2006) Diagnosis of Cirrhosis by Transient Elastography (FibroScan): A Prospective Study. Gut, 55, 403-408. http://dx.doi.org/10.1136/gut.2005.069153

[6] Colletta, C., Smirne, C., Fabris, C., et al. (2005) Value of Two Noninvasive Methods to Detect Progression of Fibrosis among HCV Carriers with Normal Aminotransferases. Hepatology, 42, 838-845. http://dx.doi.org/10.1002/hep.20814

[7] Morishima, C., Morgan, T.R., Everhart, J.E., et al. (2006) HCV RNA Detection by TMA during the Hepatitis C Antiviral Long-Term Treatment against Cirrhosis (Halt-C) Trial. Hepatology, 44, 360-367. http://dx.doi.org/10.1002/hep.21265

[8] Fontana, R.J., Sanyal, A.J., Mehta, S., et al. (2006) Portal Hypertensive Gastropathy in Chronic Hepatitis C Patients with Bridging Fibrosis and Compensated Cirrhosis: Results from the HALT-C Trial. American Journal of Gastroenterology, 101, 983-992. http://dx.doi.org/10.1111/j.1572-0241.2006.00461.x

[9] Giovanna, F., Carlo, F., Laurent, C., et al. (2015) WFUMB Guidelines and Recommendations for Clinical Use of Ultrasound Elastgraphy: Part 3: LIVER. Ultrasound in Medicine and Biology, 41, 1161-1179. http://dx.doi.org/10.1016/j.ultrasmedbio.2015.03.007

[10] Yamasaki, K., Tatoyama, M., Abirn, S., et al. (2014) Elevated Serum Levels of Wisteria Floribunda Agglutinin-Positive Human Mac-2 Binding Protein Predict the Development of Hepatocellular Carcinoma in Hepatitis C Patients. Hepatology, 60, 1563-1573. http://dx.doi.org/10.1002/hep.27305

[11] Kang, J., Kwon, H., Cho, J., et al. (2012) Comparative Study of Shear Wave Velocities Using Acoustic Radiation Force Impulse Technology in Hepatocellular Carcinoma: The Extent of Radiofrequency Ablation. Gut and Liver, 6, 362-367. http://dx.doi.org/10.5009/gnl.2012.6.3.362

[12] Xu, X., Luo, L., Chen, J., et al. (2014) Acoustic Radiation Force Impulse Elastography for Efficacy Evaluation after Hepatocellular Carcinoma Radiofrequency Ablation: A Comparative Study with Contrast-Enhanced Ultrasound. BioMed Research International, 2014, Article ID 901642, 7 p. 